\section{VP195 Using The ISSG Search Filter Resource In Health Technology Assessment}

\section{AUTHORS:}

Kath Wright (kath.wright@york.ac.uk), Julie Glanville, Carol Lefebvre

\section{INTRODUCTION:}

Information specialists and others searching for Health Technology Assessments (HTAs) can use the ISSG Search Filter resource (SFR) to identify filters to incorporate into search strategies. This can save time and effort when designing searches and create more efficient searches that retrieve fewer and possibly more relevant database records (link available here: https://sites.google.com/a/ york.ac.uk/issg-search-filters-resource/home).

What are search filters?. Search filters are collections of search terms designed to retrieve selections of records from bibliographic databases. Some filters are designed to retrieve records of specific study designs such as randomized controlled trials (RCTs) or systematic reviews; others aim to retrieve records relating to other features or topics such as the age or gender of study participants.

Search filters may be designed to be sensitive, precise or balanced between sensitivity and precision.

\section{METHODS:}

When would you use a search filter in HTA?. Search filters can be added to search strategies to limit to specific study types, for example, RCTs, mixed methods studies, systematic reviews. They can also be used when searching for other aspects of HTA such as patient views or specific age groups.

The ISSG SFR includes sections listing search filters to help identify adverse effects, aetiology, economic evaluations, health state utility values, public views, and quality of life.

\section{RESULTS:}

How are filters used?. A search filter is often used in combination with a topic search to restrict the search results to a specific type of record, for example, records reporting health state utility values or records of randomized controlled trials.

\section{CONCLUSIONS:}

Further guidance on the use of search filters can be found in the SuRe Info Search Filters chapter.

\section{VP196 Impact Of Trial Registry Search Features On Searches In CT.gov/ICTRP}

\section{AUTHORS:}

Elke Hausner (elke.hausner@iqwig.de), Marco Knelangen, Siw Waffenschmidt

\section{INTRODUCTION:}

In contrast to bibliographic databases, trial registries do not offer the option of formulating complex search queries, thus making targeted searches more difficult. However, ClinicalTrials.gov (CT.gov) and the International Clinical Trials Registry Platform (ICTRP) offer different search features that may help compensate this limitation. Our aim was to determine the importance of search features (for example, searches using synonyms or, additionally in CT.gov, automatic inclusion of further search fields) for trial registry searches.

\section{METHODS:}

We conducted a project called "Trial registry searches for studies of newly approved drugs" (1). One analysis investigated the question as to whether searches for different health conditions and interventions (new drugs) directly identified registry entries with the search terms entered or whether certain search features were responsible for this. We searched CT.gov and ICTRP for different conditions and interventions using the advanced search interface. For each search, we 